Lars P. Feld, Veronika Grimm, Wolf Heinrich Reuter

\title{
Zukunftsperspektiven sichern durch Reformen, nicht durch Schulden
}

\begin{abstract}
Die Corona-Pandemie hat die Welt mitten in einem Strukturwandel getroffen. Neben der weiter voranschreitenden Digitalisierung von Wirtschaft und Gesellschaft haben viele Staaten seit 2019 endlich ihre Klimaschutzbemühungen verstärkt. Die Konkretisierung von Klimaschutzzielen und politische Initiativen wie der Europäische Green Deal lassen wenig Zweifel daran, dass Geschäftsmodelle auf Basis fossiler Energieträger kaum eine Zukunft haben. Die bevorstehende Umstellung der Energiesysteme und der Wirtschaftsprozesse auf klimaneutrale Technologien sowie die zunehmende Digitalisierung werden die Art, wie wir wirtschaften und leben, verändern und erfordern hohe Investitionen.
\end{abstract}

Viele der heutigen Geschäftsfelder werden in einer digitalisierten und klimaneutralen Wirtschaft nicht weiterbestehen können. Wertschöpfungsketten werden sich daher zwar verlagern, aber im Gegenzug werden Zukunftsmärkte entstehen. Für Deutschland und Europa ist es entscheidend, diese neuen Geschäftsfelder auf Basis von Innovationen zu bedienen und so die globale Wettbewerbsfähigkeit zu stärken. Die Europäische Kommission

(C) Der/die Autor:in(nen) 2021. Open Access: Dieser Artikel wird unter der Creative Commons Namensnennung 4.0 International Lizenz veröffentlicht (creativecommons.org/licenses/by/4.0/deed.de).

Open Access wird durch die ZBW - Leibniz-Informationszentrum Wirtschaft gefördert.

Prof. Dr. Dr. h. c. Lars P. Feld ist Direktor des Walter Eucken Instituts, Professor für Wirtschaftspolitik an der Albert-Ludwigs-Universität Freiburg und wissenschaftliches Mitglied in der Mindestlohnkommission.

Prof. Dr. Veronika Grimm ist Professorin für Wirtschaftstheorie an der Friedrich-AlexanderUniversität Erlangen-Nürnberg und Mitglied im Sachverständigenrat zur Begutachtung der gesamtwirtschaftlichen Entwicklung (SVR).

Dr. Wolf Heinrich Reuter ist Generalsekretär des Sachverständigenrats (SVR). schätzt, dass im Zuge der Transformation bis 2030 rund 2,6 Billionen Euro an zusätzlichen privaten und öffentlichen Investitionen notwendig sein werden, um die Klimaziele zu erreichen (Europäische Kommission, 2019). Dabei sind öffentliche Investitionen gefragt, um Grundlagen für die Transformation zu legen, etwa bei der Infrastruktur, der Forschungsförderung oder im Bildungswesen. Der Großteil des Kapitals muss jedoch aus der Privatwirtschaft kommen. Im Durchschnitt der vergangenen zehn Jahre war die Relation von privaten zu öffentlichen Investitionen in Deutschland in etwa 8:1 (vgl. Abbildung 1). Dies zeigt die Dimension der Herausforderung: Auf die Mobilisierung privaten Kapitals wird es mit Blick auf die Transformation ankommen.

Die Corona-Krise hat mitten in der Transformation eindrucksvoll verdeutlicht, wie wichtig die Rolle des Staates - und hier insbesondere seine Stabilisierungsfunktion für die wirtschaftliche Entwicklung ist. Ohne die umfangreichen fiskalischen Maßnahmen und die Wirkung der automatischen Stabilisatoren wäre der Wirtschaftseinbruch ungleich schärfer ausgefallen. Die Tragfähigkeit der Staatsfinanzen wurde dabei weltweit einer Belastungsprobe ausgesetzt. Staaten waren gezwungen, sich für Unternehmenshilfen, Stützungs- und Konjunkturprogramme außergewöhnlich hoch zu verschulden. Die Spielräume, um die Herausforderungen der Pandemie fiskalisch zu adressieren, waren international jedoch sehr unterschiedlich. Diese unterschiedliche Reaktionsfähigkeit bestimmt, ob in einer Krise entschlossen, in großem Umfang und ohne externe Unterstützung fiskalpolitisch gegengesteuert werden kann. In wirtschaftlich guten Jahren sollte daher der Grundstein gelegt werden, um die Tragfähigkeit der öffentlichen Finanzen sicherzustellen. Der finanzpolitische Spielraum der einzelnen Staaten ist dabei in einer Währungsunion umso wichtiger, da den Mitgliedstaaten weniger Instrumente zur Herstellung der Tragfähigkeit zur Verfügung stehen. Insbesondere kann, anders als etwa die USA oder Japan, kein Mitgliedstaat alleine durch nationale Geldpolitik ökonomische Schocks abfedern.

Die Ansichten in der aktuellen wirtschaftspolitischen Debatte, wie es finanzpolitisch weitergehen soll, sind jedoch geteilt. Die einen befürworten eine Rückkehr zur Schuldenbremse im Jahr 2023 und argumentieren, dass ausreichend Spielraum für die Transformation und die Erholung von der Corona-Krise besteht. Die anderen fordern eine Lockerung der Schuldenbremse und schauen begeistert 
Abbildung 1

Entwicklung der privaten und öffentlichen Investitionen in Deutschland ${ }^{1}$

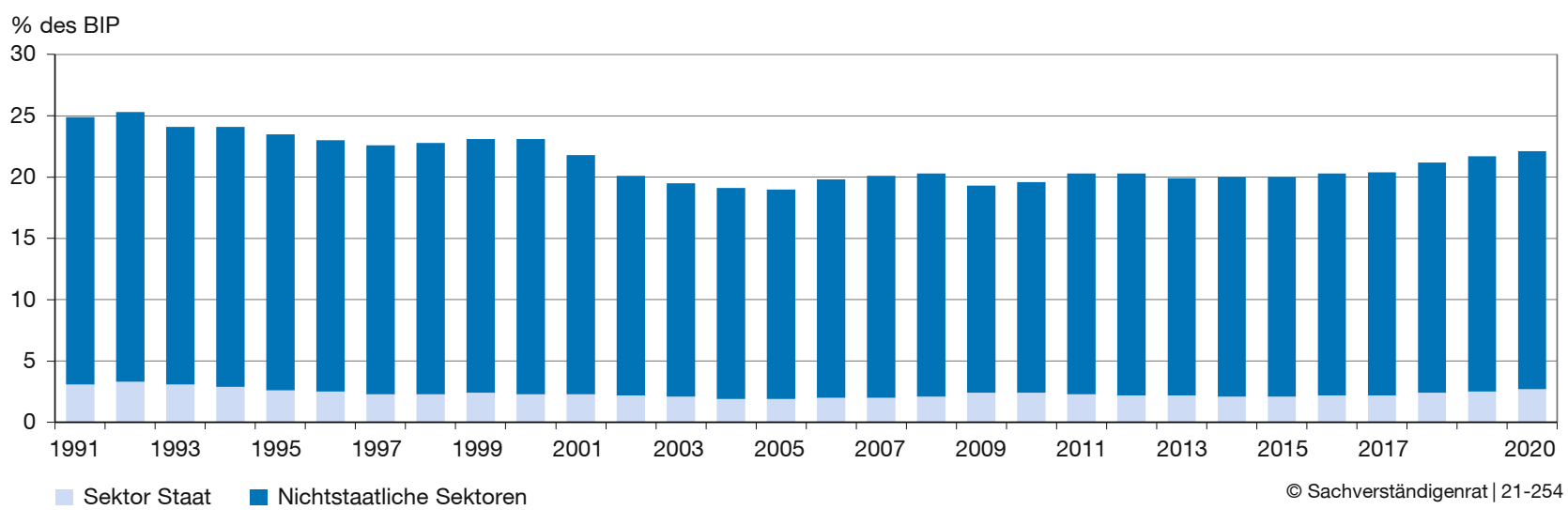

${ }^{1}$ Anteil der Bruttoanlageinvestitionen am Bruttoinlandsprodukt in jeweiligen Preisen.

Quelle: Statistisches Bundesamt.

auf die USA, wo die neue Administration unter US-Präsident Biden ein schuldenfinanziertes Wiederaufbau- und Investitionsprogramm im Umfang von jährlich ungefähr $1 \%$ des US-amerikanischen Bruttoinlandsprodukts (BIP) über die nächsten acht Jahre lanciert und mit dem Narrativ des Klimaschutzes verbindet.

Wir argumentieren im Folgenden, dass die Politik das realwirtschaftliche Umfeld und das Finanzierungsökosystem auf die zukünftigen Herausforderungen - insbesondere den Klimaschutz, die Digitalisierung und den demografischen Wandel - ausrichten sowie die verfügbaren öffentlichen Mittel zukunftsorientiert und zielgerichtet einsetzen sollte. Dabei sollten Deutschland und die EU auf fiskalische Tragfähigkeit setzen, um Wachstum in einem stabilen Umfeld zu gewährleisten. Die Schuldenbremse setzt die richtigen Anreize, zwischen gegenwarts- und zukunftsbezogenen öffentlichen Ausgaben abzuwägen und ein gutes Umfeld für private Investitionen zu schaffen. Deshalb sollte Deutschland an ihr festhalten.

\section{Die Schuldenbremse im europäischen Kontext}

Weltweit, nicht zuletzt aber in der EU, wurden Fiskalregeln eingeführt, um die öffentlichen Defizite und Schulden in wirtschaftlich normalen Zeiten zu beschränken und damit eine antizyklische Politik zu ermöglichen. Die meisten Fiskalregeln passen den fiskalischen Spielraum in normalen Zeiten dem Konjunkturzyklus an und sind mit Ausnahmeregelungen versehen, sodass sich die Staaten in Krisenzeiten umfangreich verschulden können. Dies gilt für die gesamtstaatlichen Defizit- und Ausgabenregeln der Mitgliedstaaten des Euroraums wie für die Schuldenbremse, die in Deutschland für Bund und Länder besteht. Die Schuldenbremse hat sich in der Corona-Krise bewährt.
Die Fiskalpolitik konnte ungehindert in großem Umfang auf die Krise reagieren, ohne dass die Tragfähigkeit beeinträchtigt oder Risikoprämien auf öffentliche Anleihen stark angestiegen wären.

An der Tragfähigkeit der öffentlichen Finanzen in Deutschland dürfte bei den aktuell niedrigen Zinsen auf deutsche Staatsanleihen selbst bei höheren Schuldenständen zwar kein Zweifel bestehen. Dass die Zinsen auf diesem Niveau verbleiben, ist jedoch keineswegs sicher (SVR, 2019). Bereits in den vergangenen Monaten sind die Zinsen auf zehnjährige deutsche Staatsanleihen von Dezember 2020 bis Mitte Mai 2021 unter anderem aufgrund der Erwartung höherer Inflationsraten um mehr als 0,4 Prozentpunkte angestiegen. Für stark verschuldete Mitgliedstaaten der Europäischen Währungsunion könnten höhere Zinsen die Tragfähigkeit der Staatsfinanzen infrage stellen.

Es wäre kontraproduktiv, wenn Deutschland vor diesem Hintergrund auf mehr Staatsausgaben bei höheren Defiziten setzen, von anderen Mitgliedstaaten aber Haushaltsdisziplin erwarten würde. Die Mitgliedstaaten der EU haben sich auf gemeinsame Fiskalregeln verständigt, deren Glaubwürdigkeit etwa an den Finanzmärkten davon abhängt, dass sie weder umgangen noch grundsätzlich infrage gestellt werden. Zudem dürften die Effekte auf das Wachstum in einem Mitgliedstaat durch höhere öffentliche Ausgaben in einem anderen Mitgliedstaat sehr begrenzt sein. Die Spillover-Effekte sind zu gering, als dass dies eine Aufweichung der europäischen Fiskalregeln rechtfertigen würde.

Nach der Finanzkrise war es Deutschland gelungen, die damals stark angestiegene Verschuldung innerhalb von zehn konjunkturell guten Jahren wieder abzubauen (vgl. 
Abbildung 2

Schuldenstand in Deutschland ${ }^{1}$

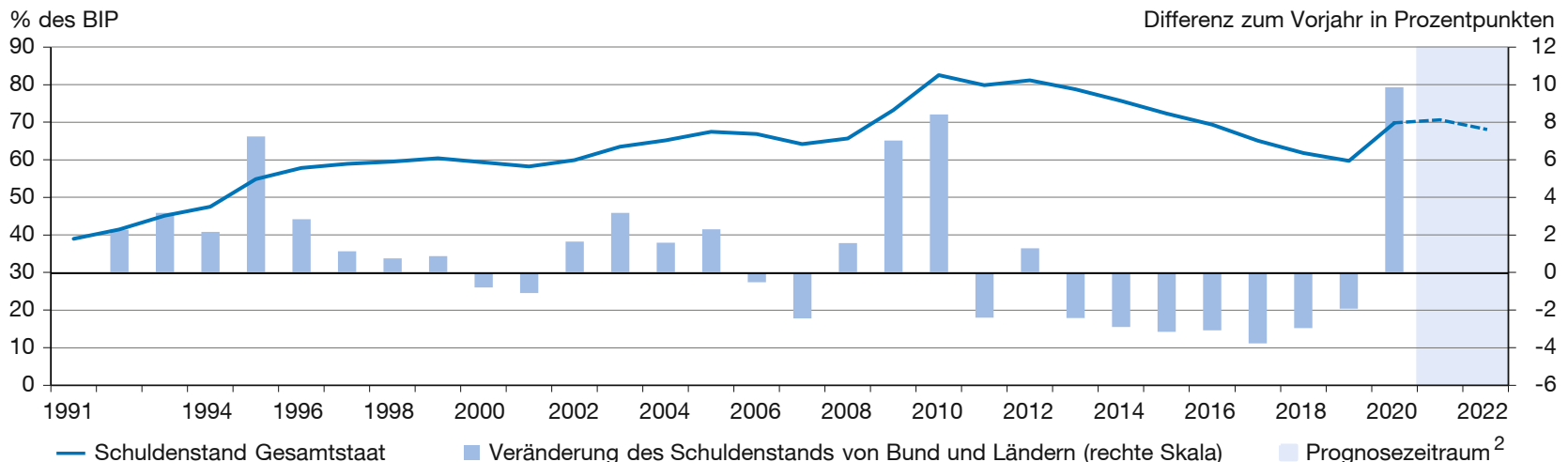

(C) Sachverständigenrat $\mid 21-253$

${ }^{1}$ Gemäß Maastricht-Abgrenzung. In Relation zum BIP. ${ }^{2}$ Prognose des Sachverständigenrates (Konjunkturprognose März 2021).

Quellen: Deutsche Bundesbank; Statistisches Bundesamt; eigene Berechnungen.

Abbildung 2). Etwas Ähnliches muss den Mitgliedstaaten des Euroraums mit hohen öffentlichen Schuldenständen nun nach der Corona-Krise ebenfalls gelingen, um für die nächste Krise vorbereitet zu sein. Dafür sind neben einer Begrenzung der Defizite vor allem Strukturreformen notwendig, die zu nachhaltigem Wachstum führen. Die Chancen für Reformen stehen besser als in den vergangenen Jahren, nicht zuletzt soweit die Zahlungen aus der europäischen Aufbau- und Resilizenzfazilität von Reformschritten begleitet werden. Das italienische Aufbau- und Resilienzprogramm stimmt dahingehend zuversichtlich. Da das „Next Generation EU“-Programm temporär und einmalig bleiben soll, müssen sich die Mitgliedstaaten darauf einstellen, die nächsten Krisen fiskalisch aus eigener Kraft zu bewältigen.

\section{Finanzpolitische Spielräume}

In den Jahren 2020 bis 2022 nimmt die Bundesregierung die Ausnahmeregelung der Schuldenbremse in Anspruch, die eine Nettokreditaufnahme über die Regelgrenze der Schuldenbremse hinaus ermöglicht. Damit dürfte die Ausnahme selbst dann bestehen, wenn 2022 aller Voraussicht nach die Wirtschaftsleistung wieder über dem Niveau von vor der Krise und nahe oder sogar über dem Produktionspotenzial liegen dürfte (SVR, 2021). Den Übergang zur Regelgrenze in den Jahren ab 2023 erleichtern 48,2 Mrd. Euro aus der Asyl-Rücklage (Feld et al., 2021) sowie zusätzlich mögliche Auszahlungen aus der europäischen Aufbau- und Resilienzfazilität, die zum Teil erst im Jahr 2023 stattfinden. Die durch die Ausnahmeregelung notwendig gewordenen Tilgungspläne, die für 2020 und 2021 zusammen derzeit 370,7 Mrd. Euro ausmachen, könnten progressiv und konjunktursensibel ausgestaltet werden, wie Feld et al. (2021) vorschlagen.
So würde die Tilgung den Spielraum der Schuldenbremse nur in wirtschaftlich guten Jahren signifikant verringern. Damit dürfte insgesamt eine Normalisierung der Fiskalpolitik und Rückkehr zur Schuldenbremse ohne eine für das Wachstum schädliche übermäßig schnelle Konsolidierung möglich sein.

Nach der Finanzkrise konnte die öffentliche Verschuldung in Deutschland insbesondere durch ein kontinuierliches und relativ hohes Wirtschaftswachstum gesenkt werden. Hinzu kommt, dass das Ausgabenwachstum (von 2011 bis 2018) unter dem Einnahmewachstum blieb (vgl. Abbildung 3). Dazu haben insbesondere sinkende Zinsausgaben beigetragen. Damit dies in den Jahren nach der Corona-Krise wieder gelingt, dürften jedoch zusätzliche Anstrengungen notwendig werden. Denn selbst wenn die Zinsen auf deutsche Staatsanleihen auf dem aktuell niedrigen Niveau verbleiben, dürfte das Potenzial für eine weitere Senkung der Zinsausgaben niedriger sein als nach der Finanzkrise. Im Zeitraum von 2012 bis 2020 sind die Zinsausgaben von $1,4 \%$ des BIP auf $0,4 \%$ des BIP gesunken. Bleiben die Zinsen auf aktuell niedrigem Niveau, dürfte der Bund im Jahr 2030 zwar sogar Zinseinnahmen in Höhe von $0,1 \%$ des BIP erzielen, die Einsparung wäre jedoch im Vergleich zu derjenigen in den vergangenen Jahren bedeutend geringer. Steigen die Zinsen um einen Prozentpunkt, ist keine weitere Absenkung im Vergleich zu 2020 zu erwarten (Feld et al., 2021). Zudem endet in den nächsten Jahren die demografische Atempause des vergangenen Jahrzehnts. Die geburtenstarken Jahrgänge stehen vor dem Renteneintritt und der Altenquotient wird deutlich ansteigen. Ohne eine Reform der gesetzlichen Renten- und Krankenversicherung werden unter anderem bei Beibehaltung der doppelten Haltelinie die Bundeszuschüsse stark ansteigen (SVR, 2020). 
Abbildung 3

Gesamtstaatliche Einnahmen und Ausgaben sowie BIP in Deutschland

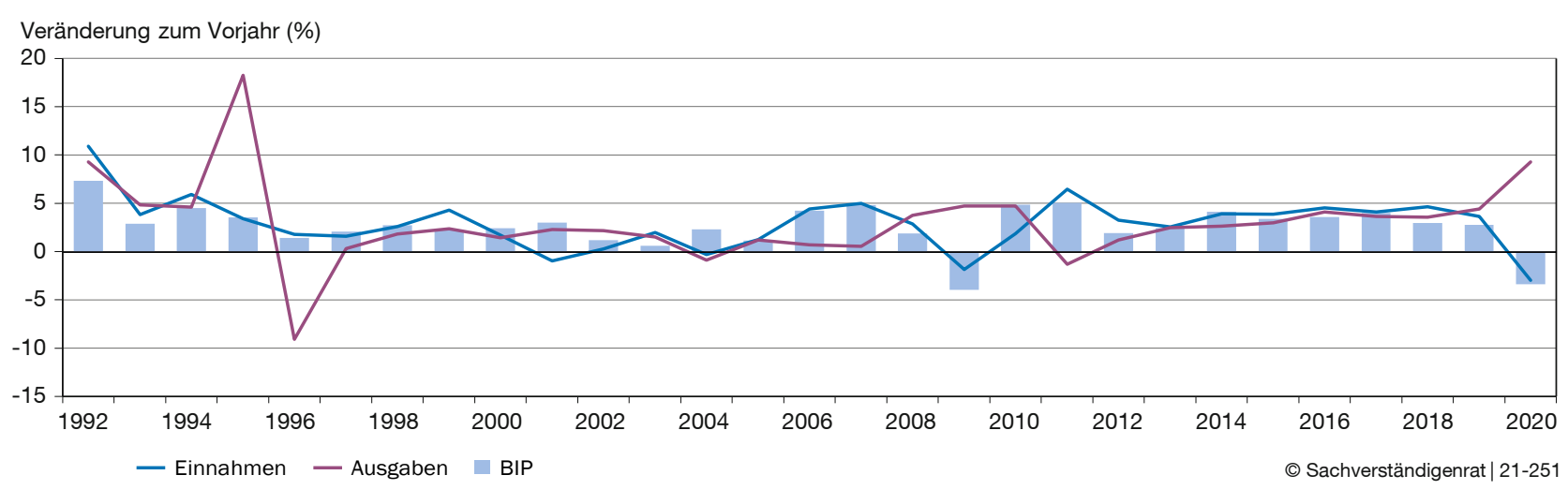

Quelle: Statistisches Bundesamt.

Um die öffentliche Verschuldung wieder zu senken, dürfte es daher insbesondere auf ein hohes und stetiges Wirtschaftswachstum in den nächsten Jahren ankommen. Wirtschaftspolitische Maßnahmen, wie diskretionäre Eingriffe, Subventionen und Steuererhöhungen, sind daher insbesondere auf deren Wirkung auf das mittelfristige Wirtschaftswachstum zu überprüfen. Es muss darum gehen, die Weichen richtig zu stellen für eine zukunftsfähige und dynamische Wirtschaft.

\section{Öffentliche Ausgaben zielgerichtet einsetzen}

In der öffentlichen Diskussion entsteht immer wieder der Eindruck, das zentrale Hemmnis für die Erreichung der Klimaziele und die Digitalisierung der deutschen Wirtschaft seien die Schuldenbremse und deren Beschränkung der öffentlichen Neuverschuldung. Es steht außer Frage, dass zusätzliche staatliche Ausgaben in diesen Bereichen notwendig sind. Vielfach sind es jedoch nicht fehlende staatliche Mittel, die der Umsetzung von öffentlichen Investitionsvorhaben entgegenstehen. Zudem fällt nicht alles, was man als Investition in die Zukunft verstehen kann, unter den Investitionsbegriff etwa der Volkswirtschaftlichen Gesamtrechnungen oder der Haushaltsordnungen.

Die Bruttoanlageinvestitionen des Bundes sind seit 2014 kontinuierlich gestiegen. Das Konjunkturpaket beinhaltet zudem zusätzliche Investitionen im Umfang von etwa 46 Mrd. Euro in den Bereichen Klimaschutz, Digitalisierung, Gesundheit und Bildung (Grimm et al., 2021). Weitere Mittel für Investitionen stehen Deutschland aus der Aufbau- und Resilienzfazilität der EU zur Verfügung. Der Finanzplan der Bundesregierung für die Jahre 2022 bis 2025 sieht Mittel in Höhe von 50 Mrd. Euro jährlich für Investitionen des Bundes vor, was eine Steigerung von rund
$30 \%$ im Vergleich zu 2019 darstellt (vgl. Abbildung 4). Das Problem in den kommenden Jahren dürfte also weniger die Höhe der auf dem Papier verabschiedeten Investitionssummen sein, sondern vielmehr die Umsetzung der Investitionsvorhaben und der zielgerichtete Einsatz der öffentlichen Mittel. Bereits seit einiger Zeit behindern die unzureichenden Kapazitäten in der öffentlichen Verwaltung, langwierige und komplizierte Genehmigungs- und Planungsverfahren sowie die Kapazitätsengpässe im Baugewerbe den Abfluss von Investitionsmitteln (SVR, 2019; Grimm et al., 2021; Wissenschaftlicher Beirat beim BMWi, 2020). Diese Hemmnisse gilt es zu beheben. Beispielsweise kann die zunehmende Digitalisierung der Verwaltung, die in der Corona-Krise Fahrt aufgenommen hat, sowohl Personalengpässe kompensieren als auch zur Beschleunigung von Arbeitsprozessen beitragen.

Um den Strukturwandel nach der Corona-Krise, den technologischen Wandel und die Transformation des Energiesystems zu adressieren, ist weit mehr als ein Anstieg öffentlicher Investitionen notwendig. So reicht es etwa nicht, jede Schule mit Breitbandzugang und Laptops auszustatten; es braucht das entsprechend qualifizierte Personal. Den bereits heute bestehenden Fachkräfteengpässen kann durch bessere Bildung, durch Umschulung und Weiterbildung begegnet werden. Auf die von der CoronaKrise ausgelösten Rückstände in der Bildung der Kinder und Jugendlichen muss mit Nachholmodellen, besserer Bildung und mehr Chancengleichheit im Bildungssystem geantwortet werden. Keine dieser Maßnahmen gilt in den Volkswirtschaftlichen Gesamtrechnungen (VGR) oder im Haushaltsrecht als Investition. Genauso wenig gelten Ausgaben zur Instandhaltung oder Investitionen von ausgelagerten Unternehmen, wie die Deutsche Bahn, als solche. Nicht nur eine Priorisierung von solchen Ausga- 


\section{Abbildung 4}

\section{Nettokreditaufnahme sowie Ein- und Ausgaben des Bundes ${ }^{1}$}

Nettokreditaufnahme des Bundes in der mittleren Frist

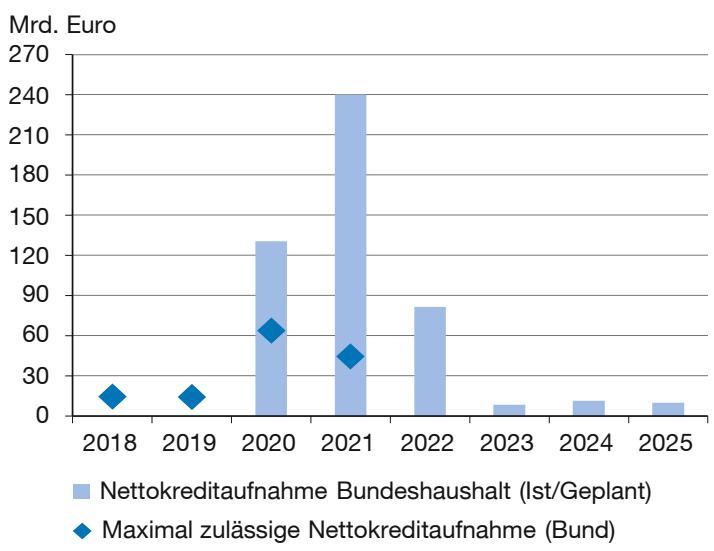

Einnahmen und Ausgaben des Bundes (Ist/Geplant) ${ }^{2}$

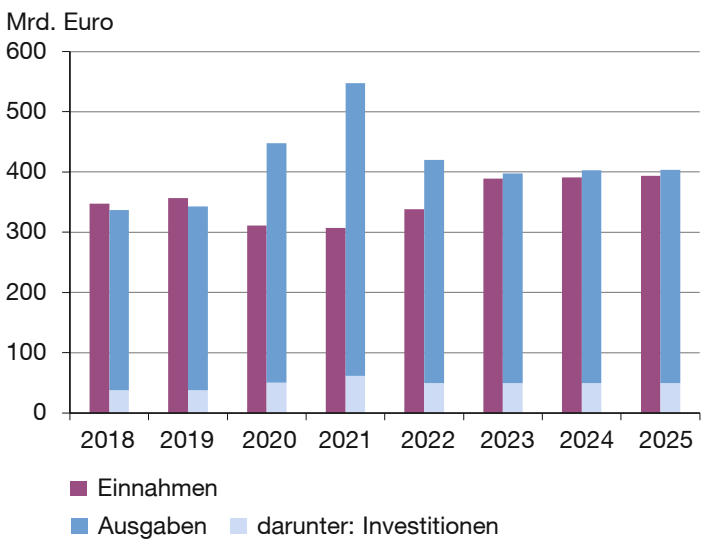

(C) Sachverständigenrat | 21-252

${ }^{1}$ Werte für 2018 und 2019 aus dem Haushaltsabschluss des jeweiligen Jahres, für 2020 aus dem vorläufigen Haushaltsabschluss vom 19. Januar 2021. Wert für 2022 aus dem Regierungsentwurf für einen Nachtragshaushalt 2021. Ab 2022 beziehen sich die Werte auf den Eckwertebeschluss der Bundesregierung zum Regierungsentwurf des Bundeshaushalts 2022 und zum Finanzplan 2021 bis 2025.

Quelle: BMF; eigene Berechnungen.

ben, die im Haushaltsrecht und in den VGR als Investitionen gelten sowie deren zielgerichteter Einsatz sind also wichtig. Vielmehr muss bei den sonstigen Ausgaben der Fokus darauf gerichtet werden, die Grundlage für zukünftiges Wachstum zu legen. Bildung und Forschung müssen hier im Mittelpunkt stehen, aber zudem Ausgaben zur Herstellung der Chancengleichheit und der sozialen Abfederung der Transformation.

\section{Privatwirtschaftliche Investitionen mobilisieren}

Öffentliche Investitionen spielen nur eine, wenngleich eine wichtige Rolle. Hingegen kann der Staat nicht die notwendigen privaten Investitionen übernehmen. Die öffentlichen Investitionen beziehen sich eher auf $\mathrm{Zu}$ schüsse zur Bereitstellung der passenden Infrastruktur, wie Breitbandnetze, Stromnetze und -speicher, oder den Ausbau von Bahn und ÖPNV. Dabei geht das Verhältnis der Größenordnungen der privaten und öffentlichen Investitionen in der öffentlichen Debatte oftmals unter. Selbst bei einer Erhöhung der öffentlichen Investitionen (für Bund, Länder und Gemeinden) um 460 Mrd. Euro über die nächsten zehn Jahre, wie etwa von Bardt et al. (2019) vorgeschlagen, würden die öffentlichen Investitionen immer noch weniger als $20 \%$ der gesamtwirtschaftlichen Investitionen ausmachen (vgl. Abbildung 1). Privatwirtschaftliche Investitionen werden die tragende Säule der bevorstehenden Transformation sein müssen. Diese wird nur mit etwas mehr staatlichen Mitteln nicht zu meistern sein.
Die Unternehmen analysieren schon heute ihre Portfolios und identifizieren Geschäftsfelder, die in Zukunft Wachstum und Wertschöpfung bringen können. Dabei ist es nicht ausgemacht, dass Investitionen in diese neuen Geschäftsfelder zwangsläufig in Deutschland oder Europa getätigt werden. Um die Investitionen hier zu mobilisieren, ist es entscheidend, die richtigen Rahmenbedingungen und Anreize für privates Handeln zu setzen und ein gutes Umfeld für private Investitionen zu schaffen. Der Staat sollte, statt den vielfältigen Rufen nach Subventionen und Förderung nachzugeben, besser die Rahmenbedingungen so anpassen, dass zukunftsorientierte Geschäftsmodelle wirtschaftlich sind. Mit Blick auf den Klimaschutz bedeutet das etwa, $\mathrm{CO}_{2}$-Preise zu stärken und verzerrende Abgaben und Umlagen konsequent abzubauen. Die Herausforderung der kommenden Jahre wird sein, diese und weitere dringend erforderliche Reformen rechtzeitig und entschlossen anzugehen. Die Gefahr ist groß, dass im Vertrauen auf die Transformationskraft staatlicher Investitionen wirkungsvolle Reformen nicht in Angriff genommen werden. So könnten sich die eigentlich erforderlichen Bemühungen weiter verzögern - bis sich das Zeitfenster im Vorfeld der nächsten Bundestagswahl wieder schließt.

Eine Stärkung der Anreize für Innovation und Investitionen gelingt insbesondere durch eine konsequente Ausrichtung des realwirtschaftlichen Umfelds auf den Klimaschutz und die digitale Transformation. Direkte und indirekte Subventionen fossiler Energieträger sollten, ebenso 
wie verzerrende Abgaben und Umlagen in der Energiebepreisung daher abgeschafft werden. Eine Stärkung der steuerlichen Forschungsförderung kann das Engagement von Unternehmen im Bereich Forschung und Entwicklung weiter stärken. Es gilt, auf eine gerechte Transformation zu achten, welche die Interessen der folgenden Generationen wahrt (im Sinne des Urteils des BVerfG) und die unteren Einkommensgruppen entlastet (z.B. durch die Entlastung der Strompreise im Gegenzug zum Anstieg der $\mathrm{CO}_{2}$-Bepreisung; SVR, 2020; SVRV, 2021).

Um Innovationen zum Durchbruch zu verhelfen und zu skalieren, ist ein guter Zugang der Unternehmen zu Wagniskapital entscheidend. Heute steht in den USA und zum Teil in Asien im Vergleich zu Europa ein Vielfaches an Wagniskapital zur Verfügung. Europa muss daher sein Finanzierungs-Ökosystem stärken und die Weichen stellen, damit Klimarisiken und -chancen an Märkten eingepreist werden können. Wichtige Komponenten sind belastbare Informationen und Indikatoren zur Nachhaltigkeit, eine echte europäische Kapitalmarktunion sowie eine Vernetzung der Expertise von Finanzwirtschaft, Realwirtschaft und Wissenschaft. Letzteres könnte die Expertise zu Nachhaltigkeitsthemen im Finanzsektor stärken, dadurch Investitionen und insbesondere mehr aktive Beteiligungen an Unternehmen auslösen. Der Ausbau der kapitalgedeckten betrieblichen Altersvorsorge könnte eine interessante Möglichkeit sein, Kapital für Zukunftsinvestitionen zu mobilisieren und gleichzeitig viele Menschen am zukünftigen wirtschaftlichen Erfolg zu beteiligen. Nicht zuletzt muss die weiter bestehende steuerliche Benachteiligung der Beteiligungsfinanzierung in den Blick genommen werden. Der Sachverständigenrat hat dazu mit seinem Modell der Zinsbereinigung des Grundkapitals einen Vorschlag unterbreitet (SVR, 2012, 2015).

\section{Die deutsche Fiskalpolitik mit den \\ Herausforderungen in Einklang bringen}

Deutschland muss seine Fiskalpolitik in den kommenden Jahren stärker darauf ausrichten, nachhaltiges Wachstum zu unterstützen und dabei sozial ausgewogen zu sein. Ob der Politik dies innerhalb der Schuldenbremse gelingen kann, wird von Kritisierenden der Schuldenbremse angezweifelt. Die polit-ökonomische Verzerrung der Finanzpolitik zu gegenwartsbezogenen Maßnahmen führe zu einer Vernachlässigung der Zukunftsausgaben des Staates und somit der investiven Komponenten der Staatstätigkeit. Daher wird die Einführung einer Goldenen Regel vorgeschlagen, welche die Investitionen von der Schuldenbremse ausnimmt, oder eines rechtlich selbständigen Sondervermögens für öffentliche Investitionen (Hüther und Südekum, 2019), sodass Investitionen über zusätzliche Schul- denaufnahme finanziert werden können und ihr Volumen von der Erhöhung anderer Ausgaben unabhängig wird.

Staatsverschuldung beschränkt zukünftige Generationen allgemein in ihrer Handlungsfähigkeit, indem sie deren fiskalischen Spielraum verkleinert. Zudem könnten die von der heutigen Generation für den öffentlichen Kapitalstock als wichtig erachteten Investitionen im Urteil zukünftiger Generationen eine geringere oder gar keine Bedeutung haben. Dabei ist fraglich, ob erforderliche öffentliche Investitionen überhaupt durch die Budgetbeschränkung der Schuldenbremse verhindert werden.

Die Bevorzugung von öffentlichen Investitionen setzt zudem ihre eindeutige Definition und die Abgrenzung zu anderen Ausgaben voraus. Gelingt dies nicht, ist die Wahrscheinlichkeit hoch, dass bei der vorgeschlagenen Änderung der Fiskalregeln lediglich mehr Spielraum für gegenwartsbezogene Ausgaben im Kernhaushalt geschaffen würde. Es würde eine Verlagerung der Diskussion drohen, die sich dann zunehmend um die Definition von Investitionen ranken würde. Eine belastbare Definition von Investitionen ist insbesondere dann schwierig, wenn sie abstrakt genug sein muss, um Bindungswirkung für alle zukünftigen Haushaltsplanungen zu haben. Der Anreiz, einen sehr weiten Investitionsbegriff zu wählen, wäre hoch. Da für zukunftsorientierte Ausgaben die Verschuldung erhöht werden könnte, gäbe es zudem weniger Hemmungen, den Befürwortenden gegenwartsbezogener Ausgaben nachzugeben - solange diese es mittragen, die Verschuldung auszuweiten, um im Kernhaushalt Platz für ihre Anliegen zu schaffen. Der Konflikt zwischen Befürwortenden gegenwarts- und zukunftsorientierter Ausgaben würde erheblich entschärft, zulasten der nachfolgenden Generationen. Es ließe sich dann nicht mehr argumentieren, dass die zusätzliche Verschuldung folgenden Generationen zugute kommt, indem sie vollumfänglich einen Gegenwert schafft.

Unter der Schuldenbremse besteht die Herausforderung einer Priorisierung von Ausgaben gleichermaßen. Doch zwingt die Budgetbeschränkung, Konflikte tatsächlich auszutragen, weil der Zielkonflikt offensichtlich ist und nicht verlagert werden kann - in der Tat wird seit Einsetzung der Schuldenbremse so intensiv über die Notwendigkeit öffentlicher Investitionen diskutiert, wie es kaum in den Dekaden zuvor der Fall war. Außerdem fällt die Zuordnung verschiedener geplanter Ausgaben in der konkreten Diskussion um die Haushaltsaufstellung deutlich leichter. So ist es z.B. schwierig, Ausgaben zur sozialen Abfederung generell als „Investition in die Zukunft“ zu begreifen. In konkreten Fällen - wie z. B. anlässlich der Corona-Krise - wäre eine solche Einordnung jedoch geboten. Statt die öffentliche Debatte um die richtige Priorisierung durch eine festgeschriebene Trennung von Ausgabenkategorien 
zu ersetzen, sollte die öffentliche Auseinandersetzung gefördert werden. Dies könnte durch eine Erhöhung der Transparenz beispielsweise über die langfristigen Auswirkungen verschiedener Ausgaben, eine Stärkung der unabhängigen fiskalpolitischen Institutionen sowie eine verstärkte öffentliche Debatte mit politischen Entscheidungstragenden erreicht werden.

Hinzu kommt, dass Bund, Länder und Gemeinden bereits in nennenswertem Umfang Investitionen außerhalb ihrer Kernhaushalte über die Extrahaushalte, über spezifische Investitionsvehikel und über ihre privatwirtschaftlichen Aktivitäten tätigen (Christofzik et al., 2019). Beim Bund dürften die Schulden der Extrahaushalte rund ein Siebtel der Bundesschulden im Jahr 2019 ausmachen. Bei den Ländern, vor allem aber bei den Kommunen dürfte dieser Anteil noch deutlich höher sein. Eine Auslagerung weiterer öffentlicher Investitionen in ein rechtlich selbständiges Sondervermögen geriete damit allmählich in Konflikt mit dem grundgesetzlich gewährten Haushaltsrecht der Parlamente. Allerdings könnten bestehende Programme der KfW für Investitionen in den Bereichen Klimaschutz und Digitalisierung vorübergehend ausgeweitet werden. Dies sollte mit einem Abbau der Beteiligungen des Staates, beispielsweise an der Commerzbank oder den Beteiligungen im Rahmen des Wirtschaftsstabilisierungsfonds (WSF), einhergehen.

Es gilt, in den kommenden Jahren mehr Spielraum für öffentliche Investitionen zu schaffen. Dazu sollten zum einen die vielfältigen Subventionen für wenig zukunftsorientierte Aktivitäten abgebaut werden. Dies beschleunigt zudem die Transformation in Richtung zukunftsorientierter Technologien. Zum anderen sollte einem weiteren überproportionalen Anstieg gegenwartsbezogener Ausgaben regelbasiert entgegengewirkt werden, sodass dieser nicht die durch das Wirtschaftswachstum entstandenen Spielräume aufbraucht. Dazu stehen etwa seit langem dringende Reformen der Sozialen Sicherungssysteme an (SVR, 2020). Insbesondere in der gesetzlichen Rentenversicherung hat sich zuletzt eine Loslösung von der Regelbindung etabliert, die es zu korrigieren gilt. So sollte die doppelte Haltelinie möglichst schon vor 2025 aufgehoben und der Nachholfaktor in der Rentenformel wieder eingesetzt werden. In der sozialen Pflegeversicherung ist ein Festhalten am Teilkaskoprinzip unabdingbar und sollte nicht durch eine Vollkaskoversicherung mit Selbstbeteiligung ersetzt werden. In der Grundsicherung müssen Bedürftigkeit und das Prinzip des Forderns und Förderns weiterhin wesentlicher Bezugspunkt bleiben.

Eine harte Budgetbeschränkung des Staates erzwingt nicht nur die Abwägung zwischen zukunfts- und gegenwartsbezogenen Ausgaben, sondern lenkt die Aufmerk- samkeit der Politik auf die Rolle privatwirtschaftlicher Investitionen für die bevorstehende Transformation. Der begrenzte fiskalpolitische Spielraum ist dabei gleichzeitig ein starker Anreiz, die dringend notwendigen Reformen anzugehen. Das Potenzial, private Investitionen durch die Ausrichtung des realwirtschaftlichen Umfelds sowie des Finanzierungsökosystems auf die Herausforderungen der Zukunft auszulösen, ist erheblich. Dies ist dringend notwendig, denn in vielerlei Hinsicht wird sich erst im Wettbewerb um die besten Lösungen entscheiden, welche Technologien sich durchsetzen und die Grundlage für Wertschöpfung und Arbeitsplätze schaffen. Die Corona-Krise hat vielfach gezeigt, dass staatliche Koordination schnell an ihre Grenzen stößt - ein Vertrauen in den „starken und lenkenden Staat“ scheint vor diesem Hintergrund nicht die logische Konsequenz. Vielmehr gilt es, auf ein gutes Zusammenspiel zwischen Staat und Privatwirtschaft zu setzen.

\section{Literatur}

Bardt, H., S. Dullien, M. Hüther und K. Rietzler (2019), Für eine solide Finanzpolitik: Investitionen ermöglichen!, IW Policy Paper, 10.

Christofzik, D. I., L. P. Feld und M. Yeter (2019), Öffentliche Investitionen: Wieviel ist zu wenig?, Schweizer Monat - Die Autorenzeitschrift für Politik, Wirtschaft und Kultur, 1064, 60-63.

Europäische Kommission (2019), Der europäische Grüne Deal, Mitteilung der Kommission an das Europäische Parlament, den Europäischen Rat, den Rat, den Europäischen Wirtschafts- und Sozialausschuss und den Ausschuss der Regionen, COM/2019/640 final, Brüssel.

Feld, L. P., L. Nöh, W. H. Reuter und M. Yeter (2021), Von der Coronabedingten Schuldenaufnahme zur Wiedereinhaltung der Schuldenbremse, Arbeitspapier, 01/21, Sachverständigenrat zur Begutachtung der gesamtwirtschaftlichen Entwicklung.

Grimm, V., L. Nöh und M. Schwarz (2021), Investitionen für nachhaltiges Wachstum in Deutschland: Status quo und Perspektiven, Wirtschaftsdienst, 101(3), 162-167, https://www.wirtschaftsdienst.eu/inhalt/ jahr/2021/heft/3/beitrag/investitionen-fuer-nachhaltiges-wachstumin-deutschland-status-quo-und-perspektiven.html (3. Juni 2021)

Hüther, M. und J. Südekum (2019), Die Schuldenbremse - eine falsche Fiskalregel am falschen Platz, Perspektiven der Wirtschaftspolitik, 20(4), 284-291.

SVR (Sachverständigenrat zur Begutachtung der gesamtwirtschaftlichen Entwicklung) (2021), Konjunkturprognose 2021 und 2022.

SVR (Sachverständigenrat zur Begutachtung der gesamtwirtschaftlichen Entwicklung) (2020), Corona-Krise gemeinsam bewältigen, Resilienz und Wachstum stärken, Jahresgutachten 2020/21.

SVR (Sachverständigenrat zur Begutachtung der gesamtwirtschaftlichen Entwicklung) (2019), Den Strukturwandel meistern, Jahresgutachten 2019/20, 238-313.

SVR (Sachverständigenrat zur Begutachtung der gesamtwirtschaftlichen Entwicklung) (2015), Zukunftsfähigkeit in den Mittelpunkt, Jahresgutachten 2015/16.

SVR (Sachverständigenrat zur Begutachtung der gesamtwirtschaftlichen Entwicklung) (2012), Stabile Architektur für Europa - Handlungsbedarf im Inland, Jahresgutachten 2012/13.

SVRV (Sachverständigenrat für Verbraucherfragen) (2021), Gutachten zur Lage der Verbraucherinnen und Verbraucher in Deutschland.

Wissenschaftlicher Beirat beim BMWi (2020), Öffentliche Infrastruktur in Deutschland: Probleme und Reformbedarf, Gutachten des Wissenschaftlichen Beirats beim Bundesministerium für Wirtschaft und Energie, Juni. 